\title{
THE SECOND VARIATION FOR VARIATIONAL PROBLEMS IN CANONICAL FORM
}

\author{
BY ROBERT HERMANN ${ }^{1}$ \\ Communicated by S. S. Chern, September 22, 1964
}

1. Introduction. This work is a sequel to [2], although it can be read independently. In the paper on which this announcement is based we develop a differential-geometric formalism for variational problems that can serve as well for multiple as for single integral problems, that does not require the introduction of local coordinate systems (which is often awkward in geometric situations) and that is well-adapted to computation. For example, we compute quite easily the second variation formula for minimal submanifolds of Riemannian spaces (which apparently is not in the literature) and can then present some geometric applications, since the geometric meaning of the terms is very clear in our formula 3.2.

The consideration of variational problems in "canonical form" (see below for the definition) leads to a description of the extremal submanifolds in terms of Cartan's theory of exterior differential systems. We will make use here of the geometric ideas and notation that were introduced in [3] for dealing with Cartan's theory.

2. The first and second variation. Let $N$ be an oriented manifold with oriented boundary $\partial N$. Let $M$ be another manifold, with $\operatorname{dim} N \leqq \operatorname{dim} M$. Let $E$ be the space of submanifold maps of $N$ into $M$. For $\phi \in E$, the "tangent space" to $E$ at $\phi$, denoted by $E_{\phi}$, is a map, typically denoted by $v$, of $N \rightarrow T(M)$ (=the tangent bundle of $M$ ) such that $v(p) \in M_{\phi(p)}$ for $p \in N$. A deformation $t \rightarrow \phi_{t}$ of $\phi$, i.e., a curve in $E$ beginning at $\phi$, defines an element $v \in E_{\phi}$ : For $p \in N, v(p)$ is the tangent vector to the curve $t \rightarrow \phi_{t}(p)$ at $t=0$. The following formula is proved in [3]:

2.1 .

$$
\left.\frac{\partial}{\partial \tau} \phi_{t}^{*}(\theta)\right|_{t=0}=\phi^{*}(v \neg d \theta)+d \phi^{*}(v \neg \theta)
$$

for each differential form $\theta$ on $M$.

In general, the calculus of variations involves the theory of critical points of a real-valued function $L$ on $E$, with $L(\phi)$ obtained by integrating a function of the derivatives of $\phi$ over. $N$. The given data for a problem in canonical form is an $r$-differential form $\theta(r=\operatorname{dim} N)$ and

${ }^{1}$ This work was supported by the Mathematics Division of the Air Force Office of Scientific Research. 
a differential ideal $I$ of differential forms on $M . L(\phi)$ is then defined as $\int_{N} \phi^{*}(\theta)$. Let $E(I)$ be the set of $\phi \in E$ that are integral submanifolds of $I$, i.e., satisfy $\phi^{*}(\omega)=0$ for all $\omega \in I$. The problem is to study the critical points of $L$ restricted to $E(I)$.

It seems that any variational problem can be prolonged to one in canonical form [4]. Note also that Lepage and his coworkers have shown that the problems in canonical form are well-adapted to discussing the "extremal field" idea in full generality.

The first variation formula follows immediately from 2.1 :

2.2 .

$$
\left.\left.\left.\frac{d}{d t} L\left(\phi_{t}\right)\right|_{t=0}=\int_{\partial N} \phi^{*}(v\urcorner \theta\right)+\int_{N} \phi^{*}(v\urcorner d \theta\right) .
$$

In order that $\phi_{t}$ lie in $E(I)$, the infinitesimal deformation $v$ should satisfy the linear variational equation

2.3 .

$$
d \phi^{*}(v \neg \omega)+\phi^{*}(v \neg d \omega)=0 \text { for all } \omega \in I .
$$

We say that $\phi \in E(I)$ is an extremal if:

2.4 .

$$
\phi^{*}(v \neg d \theta)=0 \text { for all } v \in E_{\phi} \text { satisfying 2.3. }
$$

If 2.4 is true for all $v \in E_{\phi}$, we say that $\phi$ is an extremal of the first kind. Notice that they can be described as the integral manifolds of the differential ideal generated by $I$ and the $X \neg d \theta$, where $X$ runs over the vector fields of $M$. This type of extremal gives the simplest second variation formula; $\phi \in E(I)$ will be one of this kind for the rest of the paper.

We can also read off from 2.2 the definition of transversality; $v \in E_{\phi}$ is transversal to $\phi$ at the boundary if:

$$
\left.\phi^{*}(v\urcorner \theta\right)=0 \text { when restricted to } \partial N \text {. }
$$

To find the second variation, we must compute $d^{2}\left(L\left(\phi_{t}\right)\right) / d t^{2}$ at $t=0$. If 2.5 is true, it is readily verified that the result only involves the values of $v$ on $N$, hence, in computing it, we can suppose without loss in generality that $\nabla$ is the restriction to $\phi(N)$ of a vector field $X$ on $V(M)$, such that each curve $t \rightarrow \phi_{t}(p)$ is an integral curve of $X$. Then

$$
\left.\left.\frac{d}{d t} L\left(\phi_{t}\right)=\int_{\partial N} \phi_{t}^{*}(X\urcorner \theta\right)+\int_{N} \phi_{t}^{*}(X\urcorner d \theta\right)
$$

hence

2.6. $\left.\frac{d^{2}}{d t^{2}} L\left(\phi_{t}\right)\right|_{t=0}=\int_{\partial N} \phi^{*}(X \neg d(X \neg \theta))+\int_{N} \phi^{*}(X \neg d(X \neg d \theta))$. 
It is readily verified directly now that the integrands of 2.6 only involve the derivatives of $X$ restricted to $N$.

3. Minimal submanifolds. Now suppose that $M$ is an oriented Riemannian manifold of dimension $m$. Choose the following range of indices and summation conventions: $1 \leqq a, b, \cdots \leqq r ; r+1 \leqq u, v, \cdots$ $\leqq m ; 1 \leqq i, j, \cdots \leqq m$. For $\phi \in E, L(\phi)$ is now the volume of $N$ with respect to the Riemannian metric induced by $\phi$. This can be put in canonical form in the following way: Choose an orthonormal basis $\left(\omega_{i}\right)$ for 1 -forms on $M$ such that $\phi^{*}\left(\omega_{u}\right)=0$. Let $\theta=\omega_{1} \wedge \cdots \wedge \omega_{r}$, and let $I$ be generated by the $\omega_{u}$. (Even if such a global choice of frame is not possible, the results obtained using this choice hold in general because they are independent of frame. To be completely precise, a standard sort of patching argument would be needed.) Let $\left(\omega_{i j}\right)$ be the connection forms with respect to the given moving frame, and let $\left(\Omega_{i j}\right)$ be the curvature forms.

For simplicity, we will only consider here the case where $N$ is compact. It can then be seen that the tangential component of $v$ does not contribute to the second variation, hence $v$ can be supposed a cross-section to the normal bundle of $\phi(N)$, which is denoted by $\phi(N)^{\perp}$. This bundle has a linear connection: In terms of the moving frames considered above, the connection forms are $\left(\omega_{u v}\right)$. The condition that this connection be flat is:

$$
\phi^{*}\left(\omega_{u a} \wedge \omega_{a v}+\Omega_{u v}\right)=0 .
$$

The second fundamental forms of $\phi(N)$ are $\phi^{*}\left(\omega_{u a} \cdot \omega_{a}\right)$. For example, 3.1 is satisfied if $M$ is of constant curvature and if all the symmetric transformations determined by the second fundamental forms commute.

Using this connection and the induced metric on $N$, we can define a Laplace operator $v \rightarrow \Delta v$ on cross-sections of the normal bundle. The main properties we will need are that $\int_{N}\langle\Delta v, v\rangle \phi^{*}(\theta)$ is nonpositive (always assuming $N$ compact), and zero if and only if $v$ has zero covariant derivative with respect to this connection. It can be shown, using 2.4, that $\phi$ is an extremal if and only if the traces of the second fundamental forms are zero (i.e., $\phi^{*}\left(\omega_{u 1} \wedge \omega_{2} \wedge \cdots \wedge \omega_{r}+\omega_{1} \wedge \omega_{u 2}\right.$ $\left.\wedge \omega_{3} \wedge \cdots \wedge \omega_{r}+\cdots\right)=0$ ), and that it is then an extremal of the first kind. Making 2.6 explicit now gives the second variation formula:

3.2. $\left.\frac{d^{2}}{d t^{2}} L\left(\phi_{t}\right)\right|_{t=0}=\int_{N}\left\{-\langle\Delta v, v\rangle+\|v\|^{2}(\lambda(v)-R(v))\right\} \phi^{*}(\theta)$. 
$R(v(p))$, for $p \in N$, is defined as follows: Choose any orthonormal basis of $\phi(N)_{\phi(p)}$, say $u_{1}, \cdots, u_{r} . R(v(p))$ is then $K\left(v(p), u_{1}\right)+\cdots$ $K\left(v(p), u_{r}\right)$, where $K($,$) denotes the sectional curvature (with$ respect to the metric on $M$ ) of the plane spanned by the two vectors. $\lambda(v(p))$ is defined as the sum of the squares of the eigenvalues of the second fundamental form of $\phi(N)$ in the unit direction determined by $v(p)$.

Finally, we can, because of the very simple form of 3.2, derive some immediate geometric conclusions, generalizing well-known facts in the geodesic case, i.e., $\operatorname{dim} N=1$.

If the sectional curvature of $M$ is nonpositive, then the second variation is non-negative, indicating, for example, that, generically, the minimal imbeddings of $N$ are isolated.

If the curvature of $M$ is positive, if $N$ is simply connected, if 3.1 is satisfied, and if the eigenvalues of the second fundamental forms of $\phi(N)$ are suitably majorized by the curvature of $M$, then there are imbeddings of $N$ arbitrarily close to $\phi$ which have less area.

Judging from a talk given by him at Berkeley recently, there is some relation of the results in this section to as-yet-unpublished work by J. Simons. His methods seem to be different.

Added in proof. $v\urcorner \theta$ denotes the contraction of the form $\theta$ by the vector field $v$.

\section{BiBLIOGRAPHY}

1. J. Eells and J. H. Sampson, Harmonic mappings of Riemannian manifolds, Amer. J. Math. 86 (1964), 109-160.

2. R. Hermann, Some differential-geometric aspects of the Lagrange variational problem, Illinois J. Math. 6 (1962), 634-672.

3. - Cartan's geometric theory of partial differential equations, Advances in Mathematics, Academic Press, New York (to appear).

4. T. Lepage, Sur les champs géodesiques du calcul des variations, Bull. Acad. Belg. Cl. Sci. 22 (1936), 716-729; 1036-1046.

NORTHWESTERN UNIVERSITY 